\title{
Clinical experience with metolazone in fifty-two African patients: synergy with frusemide
}

\author{
R. F. GUNSTONE* \\ B.Sc., M.B., M.R.C.P. \\ Senior Medical Registrar
}

H. G. P. Shani
D. NJEMO

\author{
A. J. Wing $\dagger$ \\ M.A., D.M., M.R.C.P. \\ Nuffield Fellow
}

E. M. W. Sabuka

\section{Medical Students \\ Medical Students \\ Department of Medicine, Mulago Hospital and Makerere University Medical School, Kampala, Uganda}

\section{Summary}

The use of metolazone (Zaroxolyn), a new diuretic, in fifty-two African patients is described. The drug was found to be safe and moderately effective when used alone, but combined with frusemide it proved to be of great value in patients whose fluid retention was refractory to other diuretics.

\section{Introduction}

Metolazone is structurally related to quinethazone. Its diuretic and hypotensive actions have been demonstrated in animals (Belair et al., 1969), and it has been found to be more potent than hydrochlorothiazide. In normal man it has been shown that diuresis occurs less promptly but is more prolonged than with frusemide (Materson \& Barkin, 1969).

We report here on our experience of metolazone in Uganda where it was used in a two part trial:

Part 1. (Patients 1-35) was a preliminary study in which the drug was given to unselected patients with oedema and/or ascites. The aim of this part of the study was to assess the safety of the drug, both

* Present appointment, Consultant Physician, Coventry Group of Hospitals, Whitley Hospital, Coventry, CV3 4AQ. † Present address: St Thomas' Hospital, London, S.E.1. clinically and by following several laboratory parameters.

Part 2. (Patients 36-52) was a further study of patients who were selected because of oedema and/or ascites resistant to conventional diuretics. Such refractory fluid retention is a common feature of clinical practice in Uganda and is encountered in the nephrotic syndrome associated with quartan malaria, in cardiac disease, especially endomyocardial fibrosis, and in hepatic cirrhosis.

In neither part of this trial were pregnant women or patients under the age of 16 given the new drug.

\section{Patients and methods}

\section{Part 1}

Thirty-five hospital in-patients with salt and water retention of diverse aetiology were studied. Brief clinical details are given in Table 1.

\section{Part 2}

Seventeen in-patients with resistant fluid retention were selected for a trial of combined treatment with metolazone and frusemide. Clinical details are given in Table 2. Their degree of resistance during the

TABle 1. Patients in Part 1 of the trial

\begin{tabular}{clcccc}
\hline \multirow{2}{*}{ Patient no. } & Diagnosis & Total & \multicolumn{3}{c}{ Result } \\
\cline { 5 - 6 } & & & Success & Failure & Indeterminate \\
\hline $1-19$ & Congestive cardiac failure & 19 & 9 & 7 & 3 \\
$20-22$ & Hepatic cirrhosis & 3 & 1 & 2 & - \\
$23-31$ & Nephrotic syndrome & 9 & 4 & 5 & - \\
$32-35$ & Miscellaneous* & 4 & 1 & 2 & 1 \\
\hline
\end{tabular}

* 32, amyloidosis; 33, chronic lymphatic leukaemia; 34, pancreatic ascites; 35, undiagnosed. 
TABle 2. Patients in Part 2 of the trial

\begin{tabular}{llll}
\hline No. & Age & Sex & \multicolumn{1}{c}{ Diagnosis } \\
\hline 36 & 58 & M & CCF, aortic incompetence, chronic renal failure \\
37 & 35 & F & $\begin{array}{l}\text { CCF, hypertension, chronic renal failure } \\
\text { CCF, mitral incompetence, endomyocardial fibrosis, } \\
\text { big spleen disease }\end{array}$ \\
38 & 29 & M & CCF, endomyocardial fibrosis, atrial flutter \\
39 & 30 & F & CCF, chronic renal failure \\
40 & 33 & M & CCF, aortic incompetence, hypertension \\
41 & 51 & M & Cor pulmonale \\
42 & 50 & M & CCF, endomyocardial fibrosis \\
43 & 20 & M & Hepatic cirrhosis \\
44 & 50 & M & CCF, endomyocardial fibrosis, atrial fibrillation \\
45 & 19 & M & Hepatic cirrhosis \\
46 & 40 & M & Hepatic cirrhosis \\
47 & 33 & M & CCF, chronic renal failure, hypertension \\
48 & 28 & M & Hepatic cirrhosis \\
49 & 40 & F & CCF, cause uncertain \\
50 & 35 & F & CCF, hypertension \\
51 & 21 & M & CCF, endomyocardial fibrosis, atrial fibrillation \\
52 & & & CCF
\end{tabular}

CCF, congestive cardiac failure.

control period of conventional treatment is illustrated in Figs. 1-3.

\section{Out-patients}

Two patients from Part 1, and ten patients from Part 2 were treated with metolazone after discharge from hospital.

The following observations were made on all in-patients:

Daily clinical examination with special reference to pulse, blood pressure and degree of fluid retention.

Daily weight, which was considered a simpler and more accurate index of fluid retention than fluid balance charts.

Routine urine testing, estimation of haemoglobin, white blood cell count, blood urea and serum electrolytes before treatment with metolazone and weekly thereafter.

On twenty-three patients serum uric acid, proteins, bilirubin, alkaline phosphatase and pyruvate transaminase were performed before and during treatment. Glucose tolerance tests were performed on two patients before and during treatment, and on two patients who developed glycosuria while on metolazone.

\section{Results}

\section{A. Diuretic effect}

If a weight loss of $0.5 \mathrm{~kg} /$ day for at least 4 days occurred, the treatment was recorded as successful.

In the thirty-five patients of Part 1 it was successful in fifteen, unsuccessful in sixteen, and in four the results were indeterminate (Table 1). Three of these four were too ill to be moved from their beds to a weighing machine, and the fourth, who had pancreatic ascites (Dreiling, 1970), lost weight on diuretics but simultaneously suffered from anorexia and repeated vomiting. Success was achieved with daily doses of metolazone of 5-150 mg but in other patients $5-100 \mathrm{mg}$ failed. Two patients responded to a combination of frusemide and metolazone, but three had no benefit from this regime.

For the seventeen patients in Part 2, selected because of their failure to respond to previof

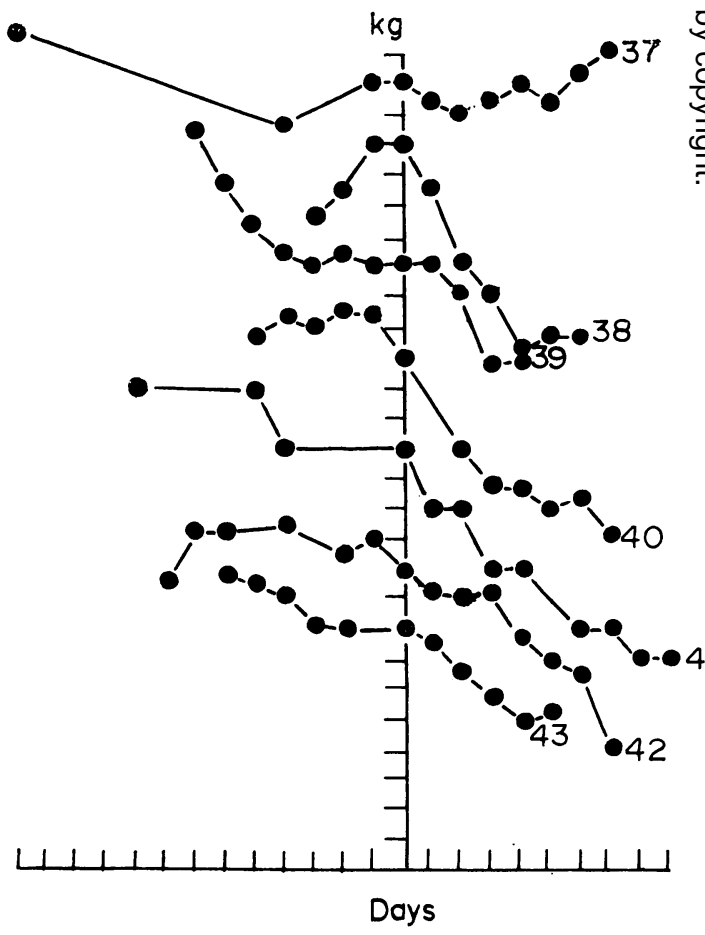

FIG. 1. Weight change in patients on adding metolazone $2.5 \mathrm{mg}$ daily to the dose of frusemide given. Daily dose of frusemide (mg): Patient 37, 240; Patient 39, 80; Patients $38,40-43,120$. The vertical line indicates the first day of metolazone. 
therapy, the combined treatment of metolazone plus frusemide was prescribed in one of three ways:

(1) Addition of metolazone to previous therapy. (Patients 37-43, Fig. 1.) Seven patients were treated in this way, and the treatment was successful in six.

Case history: Patient 38 was a cultivator, aged 29, under regular treatment for congestive cardiac failure due to endomyocardial fibrosis with mitral incompetence. He was re-admitted with ascites and left lower lobe pneumonia. The latter responded

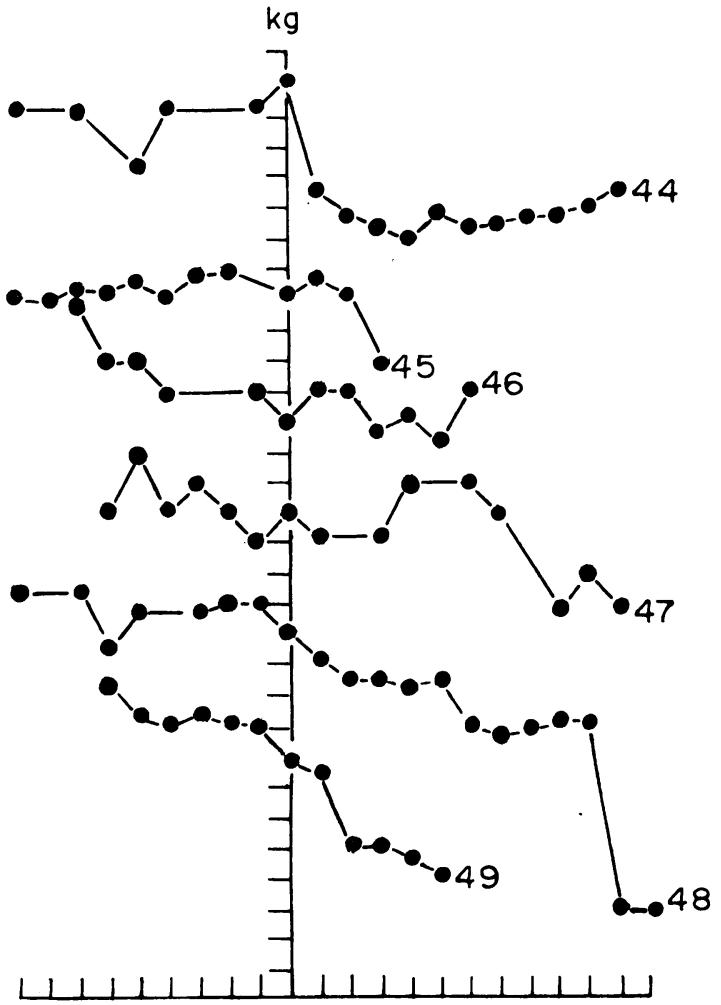

Days

FIG. 2. Weight changes in patients on substituting metolazone for spironolactone. The vertical line indicates the first day of the metolazone. (See data set out in table below.) rapidly to penicillin but despite digoxin and frusemide $120 \mathrm{mg}$ daily, his ascites increased and he steadily gained weight. On adding $2.5 \mathrm{mg}$ metolazone daily to this regime, prompt diuresis occurred, and he subsequently remained free of ascites and oedema for 85 days as an out-patient taking digoxin $0.25 \mathrm{mg}$, frusemide $80 \mathrm{mg}$ and metolazone $2 \cdot 5 \mathrm{mg}$ daily. When the combined diuretic regime was replaced by bendrofluazide, ascites recurred but control of ascites was readily regained with metolazone and frusemide.

(2) Substitution of metolazone for spironolactone. (Patients 44-49, Fig. 2.) Six patients were treated in this way, and the treatment was successful in four.

Case histories: Patient 44 who had ascites due to hepatic cirrhosis failed to lose weight on frusemide $400 \mathrm{mg}$ and spironolactone $100 \mathrm{mg}$ daily, but his weight fell promptly when metolazone $10 \mathrm{mg}$ was substituted for the spironolactone. Subsequently, it was necessary to increase the metolazone to $100 \mathrm{mg}$ daily to maintain the diuresis.

Patient 47, who also had hepatic cirrhosis and ascites, failed to respond when metolazone was substituted for spironolactone, but he did lose weight after the reintroduction of spironolactone to constitute a regime of frusemide $120 \mathrm{mg}$, metolazone $200 \mathrm{mg}$ and spironolactone $100 \mathrm{mg}$ daily.

(3) Cross over studies. (Patients 36, 50-52, Fig. 3.) In four patients metolazone and frusemide were assessed independently. Metolazone was given alone, and frusemide either alone or in combination with diuretics other than metolazone. The effect of the combination of metolazone with frusemide was then observed. In three of these patients the combination of metolazone with frusemide was clearly shown to be effective when all the other regimens had failed.

Case histories: Patient 36 had anasarca attributed to congestive heart failure secondary to aortic valve disease and chronic renal failure. The maximal dose of metolazone ( $200 \mathrm{mg}$ daily) failed to cause a diuresis as did high dose frusemide (200 mg daily) combined with spironolactone and thiazide. On adding $10 \mathrm{mg}$ of metolazone daily to this massive diuretic therapy, he became ambulant and free of oedema.

\begin{tabular}{|c|c|c|c|c|}
\hline \multirow{2}{*}{ Patient } & \multicolumn{2}{|c|}{ Treatment before metolazone (mg/day) } & \multicolumn{2}{|c|}{ Treatment after metolazone $(\mathrm{mg} / \mathrm{day})$} \\
\hline & Frusemide & Spironolactone & Frusemide & Metolazone \\
\hline 44 & 400 & 100 & 400 & 10 \\
\hline 45 & 160 & 100 & 160 & 10 \\
\hline 46 & 120 & 200 & 120 & 5 \\
\hline 47 & 480 & 100 & 220 & $2 \cdot 5-10$ \\
\hline 48 & 240 & 100 & 240 & $5-20$ \\
\hline 49 & 200 & 150 & 200 & $2 \cdot 5$ \\
\hline
\end{tabular}




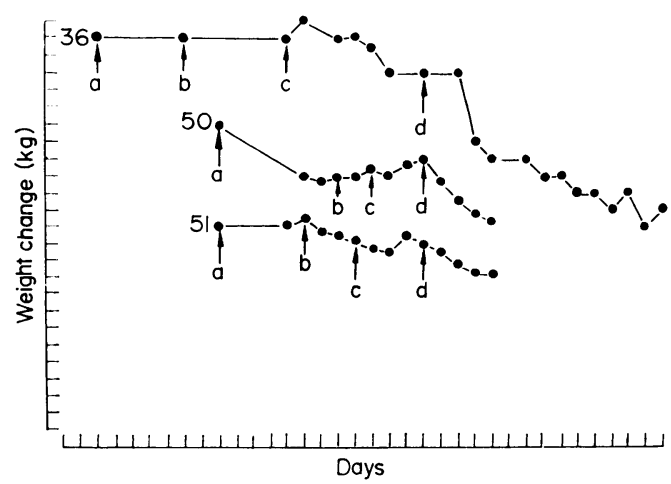

FIG. 3. Weight changes in patients 36,50 and 51 .

The arrows $a, b, c$ and $d$ indicate changes in therapy in individual patients as follows (diuretic dose in $\mathrm{mg}$ / day):

Patient 36-a, metolazone 200; b, bendrofluazide $15+$ spironolactone 100 ; c, as at b but with frusemide 200 added; $d$, as at $c$ but with metolazone 10 added.

Patient 50-a, frusemide 320; b, metolazone 10; c, metolazone 20 ; d, frusemide $120+$ metolazone $2 \cdot 5$.

Patient 51-a, frusemide 80; b, frusemide 180 ; c, meto-

lazone $50 ; \mathrm{d}$, frusemide $120+$ metolazone $2 \cdot 5$.

Patients 50 and 51 were treated initially with highdose frusemide alone, then metolazone in moderate dosage (10-50 mg daily) and finally $2.5 \mathrm{mg}$ of metolazone with frusemide $120 \mathrm{mg}$ daily (a smaller dose of frusemide than that used in the control period). A prompt diuresis occurred with combined treatment in patient 50 , but in patient 51 the result was more equivocal.

Patient 52 (not shown in Fig. 3) had a 7-year history of congestive cardiac failure due to endomyocardial fibrosis with mitral incompetence, atrial fibrillation and gross ascites which regularly recurred after paracentesis despite therapy with digoxin and diuretics in large doses, such as frusemide $120 \mathrm{mg}$ daily plus spironolactone $100 \mathrm{mg}$ daily combined with salt restriction. Paracentesis was performed about once every 10 weeks. Metolazone $200 \mathrm{mg}$ daily failed to prevent recurrence of the ascites, but on combined treatment with metolazone $2.5 \mathrm{mg}$ and frusemide $80 \mathrm{mg}$ daily, diuresis occurred. Paracentesis has not been required in the initial 18 weeks of follow up of this treatment, and the patient has remained at work.

Out-patient results. Twelve patients were discharged taking metolazone. One patient (18) took it alone, while the others took it in combination with frusemide. Nine patients were seen subsequently and over periods ranging from 42 to 126 days the diuretic effect was maintained.

\section{B. Side-effects}

The side-effects common to diuretic therapy from other drugs were encountered. The blood urea rose in nineteen patients and fell in three. The serum potassium fell below normal limits in nine patients, ڤ and such a fall was sometimes noted when spirono- $\frac{2}{\mathbb{Q}}$ lactone was given with metolazone and sometimes when potassium supplements were given. There were $\stackrel{c}{\leftarrow}$ no other changes in the laboratory investigations $\overrightarrow{\mathrm{F}}$ which could not be ascribed to the underlying disease.

All clinical occurrences noted during the trial were attributable to the patient's disease with the following exceptions:

Patient 3 with cor pulmonale complained of pain in the legs for which no cause could be found.

Patient 35 (whose diagnosis was not established) $\vec{\circ}$ had persistent though not disabling oedema despite $\overrightarrow{.}$ treatment. When being treated as an out-patient he ${ }_{\sigma}^{\omega}$ developed a depression on day 75 of metolazone $\overline{0}$ therapy. This was preceded by a throat infection. 3 His electrolytes were normal at the time of the depression, as was his white cell count.

\section{Deaths}

Ten patients died while taking metolazone or soon afterwards. Most of these had such gross disease that their deaths were not unexpected and canngt be attributed to the diuretic therapy. In the following cases, however, the deaths were unexpected.

Patient 45 died suddenly the day after a brisk diuresis which occurred on day 3 of combines frusemide and metolazone treatment. He had bee्gी digitalized. On day 1 of his treatment, his seruin potassium had been $5.0 \mathrm{mEq} / \mathrm{l}$ and as he had beefi1. on spironolactone until this time potassium supple ments were not given. Just prior to his death pulsus bigeminus was noted. His serum potassium on the day of death was $3.2 \mathrm{mEq} / 1$. This was no doubt attributable to the diuresis and one factor contribut- $\mathbb{\otimes}$ ing to his death, though he had a history of paroxysmal arrhythmias.

Patient 49 had cirrhosis of the liver manifested by oedema and ascites resistant to frusemide $200 \mathrm{mg}$ and spironolactone $150 \mathrm{mg}$ daily. Steady weight loss occurred on substituting metolazone $2.5 \mathrm{mg}$ daily 3 for the spironolactone. Seventeen days after starting combined treatment he was completely free of oedema 3 and ascites and diuretics were discontinued. The $\delta$ following day he had an upper respiratory infection,, and the next evening, while walking, he fell uncon-을 scious and convulsing. The convulsions were controlled with phenytoin and phenobarbitone, but he응 never regained consciousness. Necropsy showed micronodular cirrhosis of the liver with histological. evidence of active hepatitis. Moderate pulmonary $\tilde{O}$ oedema and some ascites were present, but the other ${ }_{W}^{N}$ organs including the brain were normal.

\section{Discussion}

Two factors are of the greatest importance in 
evaluating a new drug, namely, safety and efficacy. With regard to the former the most disturbing feature of the present study was the unexplained coma and death of patient 49 . The abrupt onset of coma was unlike hepatic encephalopathy, and the history is not reminiscent of other drug reactions. Moreover, the coma occurred $61 \mathrm{hr}$ after the last dose of metolazone, by which time all of the drug would have been excreted and certainly its diuretic action had ceased. His death may have been due to a curious 'brain syndrome' of unknown aetiology which is occasionally seen in the wards and at postmortems in Uganda. These cases resemble other states associated with coma, confusion and convulsions, namely, cerebral malaria, trypanosomiasis and viral encephalopathy. Standard investigations in life and routine microscopy of the brain at necropsy do not shown any diagnostic features but detailed histopathological studies of these idiopathic brain syndromes have not yet been made (Hutt, 1971).

The only other side-effects of note are a rise in blood urea and hypokalaemia. A rise in blood urea and fall in glomerular filtration rate consistently accompany effective diuresis, as has been pointed out recently by Jewkes, Burki \& Guz (1970), who were studying frusemide. Hypokalaemia has been thought to be an uncommon complication of diuretic therapy in Uganda because of the high potassium content of the plantain banana which constitutes the staple diet, and it is common practice not to prescribe potassium supplements with diuretics. Our results indicate that such supplements may be required with metolazone when it is either given alone or combined with frusemide. Spironolactone was not enough to prevent hypokalaemia in some of our patients. The onset of diuresis using the combined regime may be disturbingly abrupt.

With regard to the efficacy of metolazone, the success rate in Part 1 is comparable with that achieved with other diuretics in similar groups of patients. Many of those in whom metolazone was unsuccessful were refractory to other diuretics. The results in Part 2 allow comparison of the combined metolazone-frusemide treatment with the effect of large doses of conventional diuretics. It appears that the combined treatment is a particularly potent diuretic regimen.

Apparent synergy of metolazone and frusemide is suggested most clearly in the records of patients 38 , $41,43,49$ and in the cross-over studies. The clinical observation of a synergistic effect raises interesting problems concerning the action of diuretics. Frusemide enhances the maximal diuresis obtainable with thiazides but the addition of thiazide to a subject undergoing maximal frusemide diuresis has no effect (Robson et al., 1964; Hook \& Williamson, 1965). On the other hand, the animal experiments of Belair, Borelli \& Yelnosky (1969) showed that the action of metolazone was inhibited by Ex4877 which is known to inhibit thiazide diuresis but not frusemide (Ross \& Cafruny, 1963). Their conclusion that metolazone acts at the same site as thiazides is not compatible with the synergy suggested by our results. Further studies of the action and interaction of these drugs will be of interest.

\section{Acknowledgments}

We thank the Research Committee of Mulago Hospital and Makerere University Medical School for permission to carry out this trial, and the Pennwalt Corporation for providing metolazone and financial support.

We are indebted to Mr John Kyobe and Mr W. AdyeriKahwa for the laboratory investigations; and to $\mathrm{Mr}$ W. Brough of St George's Hospital Medical School for the illustrations.

Thanks are also due to Dr Hugh Oliver and Dr W. R. Billington, C.B.E., present and recent Medical Superintendents of Mengo Hospital, for allowing us to treat patients in their wards.

\section{References}

Belair, E.J., Borelli, A.R. \& Yelnosky, J. (1969) Antagonism to the diuretic action of SR 720-22 (33869). Proceedings of the Society for Experimental Biology and Medicine, 131, 327.

Belair, E.J., Kaiser, F., van Denburg, B., Borelli, A., LAWler, R., Pansevich, R. \& Yelnosky, J. (1969) Pharmacology of SR 720-22. Archives internationes de Pharmacodynamie et de Therapie, 177, 71.

Dreiling, D.A. (1970) The lymphatics, pancreatic ascites and pancreatic inflammatory disease. American Journal of Gastroenterology, 53, 119.

Hook, J.B. \& Williamson, H.E. (1965) Addition of the saluretic action of furosemide to the saluretic action of certain other agents. Journal of Pharmacology and Experimental Therapeutics, 148, 88.

HutT, M.S.R. (1971) Personal communication.

JEWKES, R.F., BURKI, N. \& GuZ, A. (1970). Observations of renal function in patients undergoing therapeutic diuresis with frusemide. Clinical Science, 38, 439.

MAterson, B.J. \& BARKIN, J.S. (1969) Personal communication.

Robson, A.O., Ashcroft, R., Kerr, D.N.S. \& Teasdale, G. (1964) The diuretic response to frusemide. Lancet, ii, 1085.

Ross, C.R. \& CAFRunY, E.J. (1963) Blockade of the diuretic action of the benzothiadiazines. Journal of Pharmacology and Experimental Therapeutics, 140, 125. 